\title{
Effect of the ceramic translucency on the long-term water sorption and solubility of resin cements
}

\author{
Ana Paula Menezes Vaz Queiroz ${ }^{1, *}$, Mariana Menezes \\ Vaz Queiroz ${ }^{1}$, Saryta Argolo², Richard Mark Foxton ${ }^{3}$, \\ Paula Mathias ${ }^{4}$, Andrea Nóbrega Cavalcanti ${ }^{5}$
}

${ }^{1}$ Dentistry Course, School of Medicine and Public Health of Bahia (BAHIANA). Dentistry Course School of Medicine and Public Health of Bahia (BAHIANA), Salvador, Bahia, Brazil.

${ }^{2}$ Department of Restorative Dentistry, School of Dentistry, Northeast Independent School (FAINOR), Vitoria da Conquista, Bahia, Brazil.

${ }^{3}$ Kings College London Dental Institute, King's College London, SE1 9RT, London, UK.

${ }^{4}$ Department of Restorative Dentistry, School of Dentistry, Federal University of Bahia (UFBA), Salvador, Bahia, Brazil.

${ }^{5}$ Dentistry Course, School of Medicine and Public Health of Bahia (BAHIANA), Salvador, Bahia, Brazil, and School of Dentistry, Federal University of Bahia (FOUFBA), Salvador, Bahia, Brazil.

Corresponding author: Ana Paula Menezes Vaz Queiroz (iD) https://orcid.org/0000-0002-0329-3078 Dentistry Course School of Medicine and Public Health of Bahia.

Telephone: (71) 99210-4297.

E-mail: anapaulavaz.odonto@outlook.com

Received: July 11, 2019

Accepted: December 02, 2019

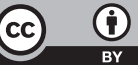

Aim: The aim of this study was to evaluate the influence of the translucency of ceramic on water sorption and solubility of resin cements over time. Methods: Lithium disilicate ceramic slides $(15 \times 15 \times 1 \mathrm{~mm}), \mathrm{A} 1$ in color and with different translucencies (high-medium-low) were manufactured; and a glass slide with similar dimension was used as control. Under every slide, 15 specimens $(8 \times 0.5 \mathrm{~mm})$ from each resin cement were prepared: light-cured (RelyX Veneer); conventional dual-cured (RelyX ARC); and self-adhesive dual-cured (Rely-X U200). The specimens were then sub-divided according to the period of evaluation (immediately, after 6 and 12 months of storage) $(n=5)$. To evaluate the loss or gain of mass, the specimens were dried until a constant mass was reached. Subsequently, they were divided according to the respective period of storage at $37^{\circ} \mathrm{C}$ in distilled water and weighed immediately following removal from water. After each period, specimens underwent a new dehydration. Values from water sorption and solubility were calculated and statistically analyzed (Anova 3-way/Tukey test). Results: The lower translucency resulted in greater water sorption and solubility for all cements, regardless of the experimental period. The self-adhesive dual-cured cement showed higher water sorption under all experimental periods and had worse values after 1 year aging. Differences among solubility could only be detected after aging, and the light-cured material had higher values after 6 and 12 months. Conclusions: It could be concluded that the low degree of translucency can negatively influence the passage of light and interfere on the durability of the resin cement. The conventional dual-cured resinous agent seemed to be less affected by such condition.

Keywords: Ceramics. Dental restoration, permanent. Resin cements. Solubility. 


\section{Introduction}

Lithium disilicate glass ceramics are highly esthetic materials that have different levels of translucency for reproducing the optical effects from natural teeth and for masking possible discolorations of the substrate ${ }^{1}$. However, for attaining successful and long-lasting restorations, such materials rely on a strong and durable cementation protocol ${ }^{2,3}$.

In adhesive cementation of glass ceramic surfaces, light-activated or conventional dualcured resin cements are frequently used. Particularly, by their capacity for adhesion to dental tissues, and by their improved mechanical properties, when compared with other agents ${ }^{4,5}$. In addition to these, self-adhesive dual-cured cements have been introduced on the market, in an endeavor to simplify the clinical steps and minimize the working time ${ }^{4}$.

The polymerization process from dual-cured resin cements occurs by two means: physical, through the action of the light on the photoinitiators; and chemical, through the reaction of benzoyl hydrogen with the tertiary amines ${ }^{6,7}$. Whereas light activated resin cements depend on the visible light of an efficient photoinitiator system so that polymerization of the material occurs effectively, and the properties of the material can be attained to the maximum extent ${ }^{5}$.

Inadequate polymerization of resin cements may be related to an insufficient quantity of light radiation that passes through the restorative material and activates the monomers, as a result of the thickness and opacity of the ceramic restoration, ${ }^{5,89}$. Therefore, the optical characteristics of materials, such as the refractive index and translucency, may determine the quantity of light transmitted, and consequently, the degree of conversion of resin cements ${ }^{5,10}$.

Long term permanence in a humid environment such as the oral cavity may also have an influence on the dynamics of diffusion of resin cements 9,10 . This may be proved by measuring the water sorption and solubility of materials, which are important tools for predicting the clinical behavior, and particularly the stability of adhesively cemented restorations ${ }^{11-14}$. However, it is important to consider that the durability and stability of the material are primordial characteristics, therefore more in depth information is obtained by means of long term studies.

In view of the foregoing, the experimental hypothesis tested by the present study was that the translucency of the ceramic could interfere in the polymerization of cements with different forms of activation (light or dual) changing their loss and gain of mass in the long term. Therefore, the aim of the present study was to evaluate the water sorption and solubility of light activated or dual-cured resin cements used under the surfaces of ceramics with different levels of translucency, in different time intervals (immediate and after 6 and 12 months of storage in distilled water).

\section{MATERIALS AND METHODS}

\section{Experimental design}

Main factors

- Ceramic translucency (4 levels): high (HT), low (LT), medium (MO) and glass slide (negative control) 
- Resin cement (3 levels): light-cured (RelyX Veneer); conventional dual-cured (RelyX ARC); and self-adhesive dual-cured (Rely-X U200)

- $\quad$ Time interval (3 levels): immediate; 6 months and 12 months

Sample: 180 resin cement specimens $(n=5)$

Dependent variables: water sorption and solubility

\section{Test specimen preparation}

In accordance with the manufacturer's recommendations, lithium disilicate ceramic (IPS e-max press, Ivoclar-Vivadent, Schaan, Liechtenstein) slides were fabricated. Briefly, this process included waxing-up the plates, sprueing, investing, pressing the respective ingots, divesting and finishing. Ceramic slides measured $1.0 \mathrm{~mm}$ thick, $15 \mathrm{~mm}$ high and $15 \mathrm{~mm}$ long, were A1 in shade, and had variation in translucency: high (HT), low (LT) and medium (MO). A glass slide, without any opacity, and of similar dimensions was used as negative control.

For preparation of resin cement specimens, a polyvinyl siloxane mold (mA) (Elite $\mathrm{H}$-D+ PuttySoft Normal Setting, Badia Polesine, Rovigo, Italy) was made. The mold had an internal orifice measuring $0.5 \mathrm{~mm}$ thick and $8 \mathrm{~mm}$ in diameter and was used for accommodating the cement.

Another polyvinyl siloxane mold (mB) was fabricated for embracing the ceramic slide, leaving just an upper orifice of 8-mm diameter. The function of this device was to prevent both the dissipation of light at the time of polymerization and the interference of external light ${ }^{8}$.

Materials with similar color (A1/Light Yellow) were selected. All the cementation agents were investigated in association with the different levels of translucency, in three experimental time intervals $(n=5)$, as described in Figure 1.

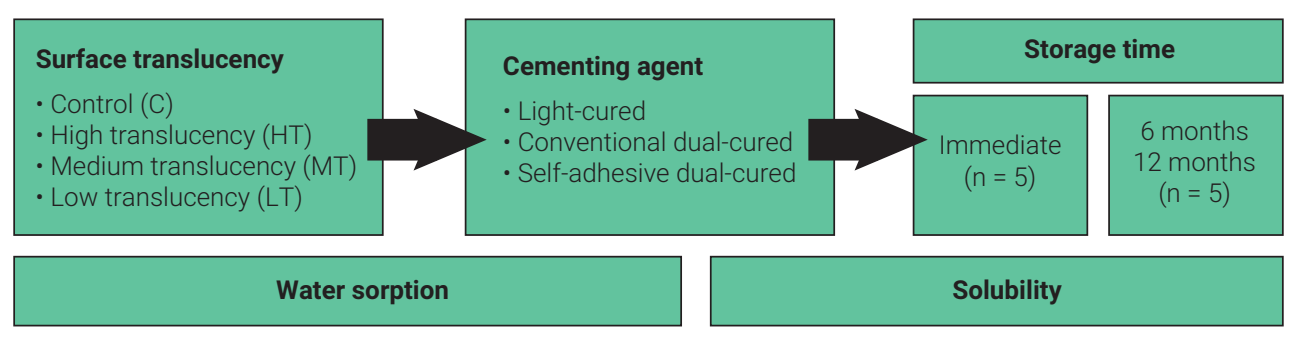

Figure 1. Distribution of Groups for water sorption and solubility tests.

The cementation agents and their formulations are described in Table 1. To prepare each specimen, the cementation agent was inserted into the first mold (mA), and a polyester strip was placed over it to accommodate the material and maintain a smooth and uniform surface. After this, a ceramic slide was placed on the above-mentioned assemble, with the second mold (mB) on top of it. A glass slide was used to exert pres- 
sure on this to force out the excess material. After removing the glass slide, polymerization was performed with a light emitting diode (LED) unit (Radii Plus, SDI, Victoria, Australia) for an exposure time of 60 seconds, with an intensity of 1,500 mW/ $\mathrm{cm}^{2}$ wavelength of $470 \mathrm{~nm}$ (Figure 2).

Table 1. Materials used and their compositions

\begin{tabular}{|c|c|}
\hline Material & Composition \\
\hline $\begin{array}{l}\text { Emax (IPS e-max, Ivoclar/ } \\
\text { Vivadente r, Schaan, Principality of } \\
\text { Liechtenstein) }\end{array}$ & $\begin{array}{l}\text { Lithium Dioxide, phosphorous oxide, aluminum, potassium oxide, and } \\
\text { other components, that are combined to produce cast glass. }\end{array}$ \\
\hline $\begin{array}{l}\text { Dual-cured resin cement, RelyX } \\
\text { ARC ( } 3 \text { M ESPE Dental Products. St. } \\
\text { Paul, MN, USA). }\end{array}$ & $\begin{array}{c}\text { Bis-GMA and TEGDMA Monomers, Zirconium/silica particle with mean } \\
\text { particle size of } 1.5 \mu \mathrm{m} \text {, Paste A: Pigments and Tertiary Amine. } \\
\text { Paste B: Benzyol Peroxide. } \\
\text { Filler Percentage of } 68 \% \text { by weight. }\end{array}$ \\
\hline $\begin{array}{l}\text { Light polymerized resin cement, } \\
\text { RelyX Veneer (3M ESPE Dental } \\
\text { Products. St. Paul - USA). }\end{array}$ & $\begin{array}{c}\text { Bis-GMA and TEGDMA Monomers Zirconium/silica particles and } \\
\text { colloidal silica Mean particle size of } 0.6 \mathrm{~mm} \\
\text { Filler Percentage of } 66 \% \text { by weight. }\end{array}$ \\
\hline $\begin{array}{l}\text { Self-adhesive dual-cured resin } \\
\text { cement, RelyX U200 (3M ESPE } \\
\text { Dental Products. St. Paul, MN, } \\
\text { USA). }\end{array}$ & $\begin{array}{l}\text { Bis-GMA and TEGDMA Monomers Inorganic particles with } 70 \% \\
\text { by weight and particle size of approximately } 12.5 \mu \mathrm{m} \text {. Base Paste: } \\
\text { Glass fiber, methacrylate phosphoric acid esters, triethyleneglycol } \\
\text { dimethacrylate, silica treated with silane and sodium persulphate. } \\
\text { Catalyzer paste: Glass fiber, substitutive dimethacrylate, silica treated } \\
\text { with silane, sodium P-toluenosulphate and calcium hydroxide. }\end{array}$ \\
\hline
\end{tabular}

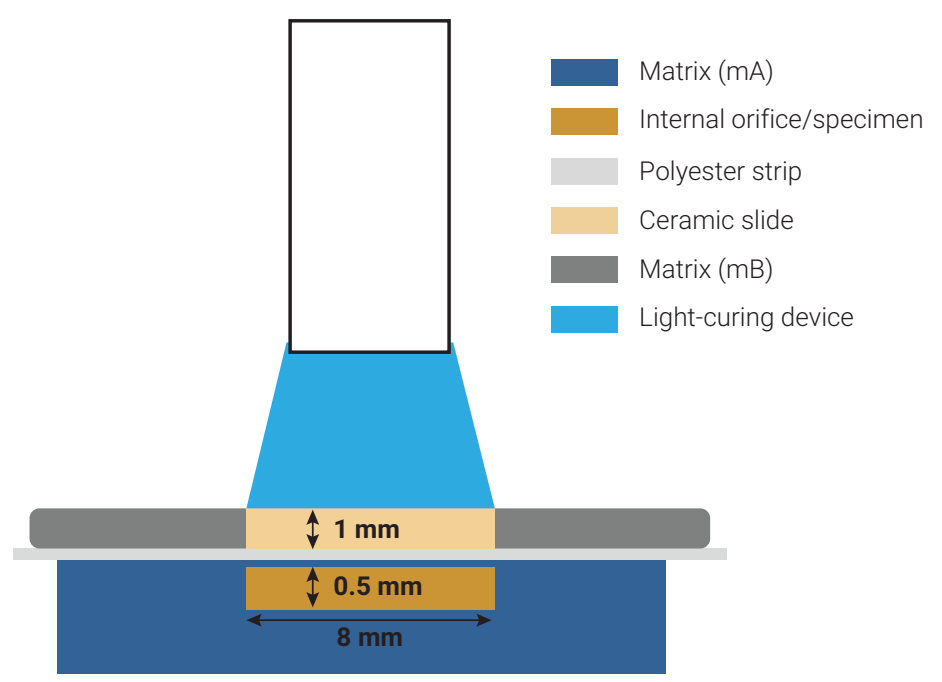

Figure 2. Illustrative diagram of test specimen fabrication.

\section{Water Sorption and Solubility Evaluation}

After been removed from the mold, the specimens were individually placed in a dark environment to prevent additional polymerization until they were ready for the water sorption and solubility test, based on the ISO 4049 specifications: 20007. Immediately 
after polymerization of the test specimens, the thickness of the resin cement cylinders was measured with a digital caliper, with precision of $0.01 \mathrm{~mm}$, and these values were used to calculate the volume $(V)$ of each unit $\left(\mathrm{mm}^{3}\right)$. Later, these specimens were individually placed in Eppendorf flasks, in a desiccator containing silica gel and then transferred for preconditioning in an oven at $37^{\circ} \mathrm{C}$.

The specimens were repeatedly weighed at intervals of 24 hours until the constant mass (M1) was obtained (variation of less than $0.2 \mathrm{mg}$ over a period of 24 hours). The weighing procedures were performed on an analytical balance (Analytical Plus, Ohaus ${ }^{\circledR}$ Corporation, Florham Park, Switzerland), with precision of one hundredth of a thousandth of a gram. After stabilization of the mass in $\mathrm{M} 1$, the groups were subdivided $(n=5)$ according to storage time in $2 \mathrm{ml}$ of distilled water $\left(\mathrm{pH} \mathrm{7.2)}\right.$ at $37^{\circ} \mathrm{C}$ : immediate analysis (7 days) or storage (6 and 12 months). After the respective time interval, the test specimens were removed from storage; washed in running water; excess was removed with absorbent paper until water was no longer visualized, and the weight was noted (M2). After weighing, the test specimens were put back into dry Eppendorf vials and put into a desiccator containing silica gel in an oven at $37^{\circ} \mathrm{C}$ to eliminate absorbed water. The specimens were weighed daily until the constant mass (M3) was obtained (variation of less than $0.2 \mathrm{mg}$ over a period of 24 hours). Water sorption (So) and solubility (Sol) in 7 days, 6 months and 12 months of storage in water were calculated using the following formula:

$$
\text { So }=(m 2-m 3) / V \quad S o l=(m 1-m 3) / V
$$

Where M1 was the mass of the sample in $\mu \mathrm{g}$ before immersion in distilled water; M2 was the mass of the sample in $\mu \mathrm{g}$ after immersion in distilled water for 7 days; 6 months and 12 months; M3 was the mass of the sample in $\mu \mathrm{g}$ after being conditioned in a desiccator with silica gel and $\mathrm{V}$ is the volume of the sample in $\mathrm{mm}^{3}$.

\section{STATISTICAL ANALYSIS}

Exploratory analysis of the water sorption and solubility data was performed to verify the homogeneity of the variances and determine whether the experimental errors presented normal distribution. Inferential statistical analysis was performed by using 3-way ANOVA and the Tukey post-hoc test for multiple comparisons of the measures, by means of the statistical program SAS, version 9.1, with a level of significance of $5 \%$. The measurement of water sorption and solubility occurred in an independent sample over time.

\section{RESULTS}

Statistical analysis of the data pointed out significant double interaction between the main factors "resin cement" and "time", both in water sorption ( $p=0.00015)$ and in solubility $(p=0.00019)$. The other statistical interactions between main factors were not considered significant ( $p>0.05$ ). Furthermore, statistically significant differences were observed among the levels of the factor "ceramic translucency" in both variables (water sorption $-p=0.00001 /$ solubility $-p=0.0005$ ). Therefore, the effect of the main factor "ceramic translucency" was tested irrespective from the other ones. 
Table 2 presents the result of statistical analysis of the water sorption data. According to this analysis, it was verified that regardless of the ceramic level of translucency, in all the storage time intervals, the resins cements presented statistical differences among them, with the highest values being presented by the self-adhesive dual-cured cement, followed by the light activated, and conventional dual-cured cement.

Furthermore, all the cements tested presented statistically higher mean values after 6 months. However, the difference in water sorption obtained between the time interval of 6 months and 1 year was not statistically significant for both the conventional dualcured resin cement and the light activated type. On the other hand, the self-adhesive dual-cured resin cement showed statistically higher water sorption values after 1 year.

Table 2. Mean (standard deviation) of water sorption data in experimental groups

\begin{tabular}{|c|c|c|c|c|c|}
\hline \multirow{2}{*}{ Ceramic } & \multirow{2}{*}{ Resinous Cement } & \multicolumn{3}{|c|}{ Time } & \\
\hline & & Initial & 6 months & 1 year & \\
\hline \multirow{3}{*}{$\begin{array}{l}\text { Control } \\
\text { (C) }\end{array}$} & Conventional Dual & $20.8(1.0) \mathrm{Cb}$ & $28.1(1.3) \mathrm{Ca}$ & $30.5(4.0) \mathrm{Ca}$ & \multirow{3}{*}{ * } \\
\hline & Light activated & 27.6 (1.4) Bb & $34.2(1.9) \mathrm{Ba}$ & 34.2 (6.7) Ba & \\
\hline & Self-adhesive Dual & $40.2(1.8) \mathrm{Ac}$ & 54.0 (1.2) Ab & $58.9(6.0) \mathrm{Aa}$ & \\
\hline \multirow{3}{*}{$\begin{array}{l}\text { High Translucency } \\
\text { (HT) }\end{array}$} & Conventional Dual & $21.7(3.6) \mathrm{Cb}$ & $27.6(4.4) \mathrm{Ca}$ & $29.0(3.2) \mathrm{Ca}$ & \multirow{3}{*}{ * } \\
\hline & Light activated & $22.3(3.0) \mathrm{Bb}$ & $30.1(5.6) \mathrm{Ba}$ & $32.8(4.7) \mathrm{Ba}$ & \\
\hline & Self-adhesive Dual & $42.3(3.0) \mathrm{Ac}$ & $50.2(0.3) \mathrm{Ab}$ & $52.8(4.7) \mathrm{Aa}$ & \\
\hline \multirow{3}{*}{$\begin{array}{l}\text { Medium } \\
\text { Translucency } \\
\text { (MO) }\end{array}$} & Conventional Dual & $20.5(1.1) \mathrm{Cb}$ & $27.0(1.7) \mathrm{Ca}$ & $25.8(1.2) \mathrm{Ca}$ & \multirow{3}{*}{ * } \\
\hline & Light activated & 26.4 (1.3) Bb & $28.0(0.2) \mathrm{Ba}$ & 30.5 (3.6) Ba & \\
\hline & Self-adhesive Dual & 41.4 (1.4) Ac & $51.2(3.4) \mathrm{Ab}$ & 54.4 (3.5) Aa & \\
\hline \multirow{3}{*}{$\begin{array}{l}\text { Low Translucency } \\
\text { (LT) }\end{array}$} & Conventional Dual & $23.0(2.8) \mathrm{Cb}$ & $29.3(1.8) \mathrm{Ca}$ & $30.2(2.2) \mathrm{Ca}$ & \multirow{3}{*}{ \# } \\
\hline & Light activated & $32.3(4.2) \mathrm{Bb}$ & $47.3(6.5) \mathrm{Ba}$ & $45.9(5.6) \mathrm{Ba}$ & \\
\hline & Self-adhesive Dual & 43.9 (4.6) Ac & 59.6 (5.2) Ab & $61.2(3.9) \mathrm{Aa}$ & \\
\hline
\end{tabular}

Different letters or symbols represent statistically significant differences (3-way ANOVA / Tukey, Alpha=5\%). Lower case letters used to compare levels of factor time for each ceramic/cement. Capital letters were used to compare cements at each level of ceramic/time. Symbols used to compare differences among ceramics. Variation Coefficient $=9.5 \%$

Relative to the effect of ceramic translucency, it was observed that irrespective of the cement tested and time interval, the highest water sorption values were noted when the low translucency ceramic was used. The results obtained with the medium and high translucency level ceramics and the control group were statistically similar among them.

Table 3 shows the results obtained by analysis of the solubility data. The differences between the resin agents was only noted after storage in water for 6 or 12 months, because in the initial period, all the resin agents presented statistically similar mean values. However, after 6 months and 1 year of storage, the light activated resin cement 
presented higher solubility values compared with those of the other materials. The effect of storage in water on the solubility of the three materials was similar; all presented statistically different mean values in the time intervals evaluated, with the highest values verified after 1 year of immersion.

Similarly to the water sorption data, the highest solubility values were associated with the surface that had low translucency. However, the surface with a mean level of opacity could also interfere in the solubility results, by presenting an intermediate effect when compared with the values verified between the surfaces with low translucency, control and high translucency.

Table 3. Mean (standard deviation) of solubility data in experimental groups

\begin{tabular}{|c|c|c|c|c|c|}
\hline \multirow{2}{*}{ Ceramic } & \multirow{2}{*}{ Resinous Cement } & \multicolumn{3}{|c|}{ Time } & \\
\hline & & Initial & 6 months & 1 year & \\
\hline \multirow{3}{*}{$\begin{array}{l}\text { Control } \\
\text { (C) }\end{array}$} & Conventional Dual & $2.9(0.1) \mathrm{Ac}$ & 4.8 (1.3) Bb & $14.9(2.9) \mathrm{Ba}$ & \multirow{3}{*}{ * } \\
\hline & Light activated & $6.2(0.1) A c$ & $10.2(5.3) \mathrm{Ab}$ & $15.2(4.2) \mathrm{Aa}$ & \\
\hline & Self-adhesive Dual & $3.2(0.1) A c$ & 8.3 (1.3) Bb & $8.1(3.4) \mathrm{Ba}$ & \\
\hline \multirow{3}{*}{$\begin{array}{l}\text { High Translucency } \\
\text { (HT) }\end{array}$} & Conventional Dual & $2.7(0.2) A c$ & $6.1(1.2) \mathrm{Bb}$ & 12.6 (2.3) Ba & \multirow{3}{*}{ * } \\
\hline & Light activated & 4.7 (2.6) Ac & 14.0 (0.9) Ab & $21.0(5.0) \mathrm{Aa}$ & \\
\hline & Self-adhesive Dual & $2.8(0.1) A c$ & 7.3 (3.0) Bb & 10.4 (4.2) Ba & \\
\hline \multirow{3}{*}{$\begin{array}{l}\text { Medium } \\
\text { Translucency } \\
\text { (MO) }\end{array}$} & Conventional Dual & 3.4 (1.2) Ac & 7.0 (1.0) Bb & 13.8 (3.5) Ba & \multirow{3}{*}{ *\# } \\
\hline & Light activated & $3.4(0.1) A c$ & $16.4(1.5) \mathrm{Ab}$ & $23.6(3.2) \mathrm{Aa}$ & \\
\hline & Self-adhesive Dual & $5.0(1.2) A c$ & 9.4 (3.7) Bb & 15.7 (7.3) Ba & \\
\hline \multirow{3}{*}{$\begin{array}{l}\text { Low Translucency } \\
\text { (LT) }\end{array}$} & Conventional Dual & $6.0(0.5) \mathrm{Ac}$ & 10.9 (2.5) Bb & $16.0(5.6) \mathrm{Ba}$ & \multirow{3}{*}{ \# } \\
\hline & Light activated & 8.1 (1.1) Ac & 16.8 (3.3)Ab & 20.1 (1.7) Aa & \\
\hline & Self-adhesive Dual & 6.0 (1.2) Ac & 11.9 (6.4) Bb & $15.5(10.0) \mathrm{Ba}$ & \\
\hline
\end{tabular}

Different letters or symbols represent statistically significant differences (3-way ANOVA / Tukey, Alpha=5\%). Lower case letters used to compare factor time for each ceramic/cement. Capital letters used to compare levels of cements at each level of ceramic/time. Symbols used to compare differences among ceramics Variation Coefficient $=31.9 \%$

\section{DISCUSSION}

Inadequate polymerization of the resin cement may be related to decreased longevity of ceramic restorations, greater stress at the interface with the dental structure; incomplete conversion of monomers into polymers, and impaired properties of the material ${ }^{9}$. Ceramic restorations in the oral medium are in constant contact with humidity, and in many cases, close to the region of the gingival sulcus. In these cases, water can act as a plasticizer, decreasing the mechanical strength and dimensional stability of the resin material, which could result in the degradation of polymers in the long term ${ }^{12}$. The experimental hypothesis of the present study was verified, since it proved that the resin cements polymerized under the ceramics with a low level of translucency presented higher levels of loss and gain of water, and that this effect was directly related to the type of material and time of storage. 
The time of immersion in the aqueous medium may affect the absorption of water and solubility of the resin materials ${ }^{13,14}$. In time-following, all the cementation agents tested presented higher values of water sorption and solubility. The composition of the organic matrix of the resin cements was based on a Bis-GMA (bisphenol A-diglycidyl dimethacrylate) or UDMA (urethane dimethacrylate) matrix in combination with other monomers with lower molecular weight, such as TEGMA (triethyleneglycol dimethacrylate). The latter promotes the formation of more hydrophilic matrices in comparison with other monomers such as Bis-EMA (bisphenol A-ethoxylate dimethacrylate), which may have allowed a higher level of diffusion dynamics of the cements tested ${ }^{13,15}$. In a similar manner, Aguiar et al in $2014^{14}$, conducted a study comparing the water sorption and solubility of five resin cements of different commercial brands, in two experimental time intervals (24 hours and 7 days). The authors concluded that time has an influence on the durability of the cements, because the presence of hydrophilic monomers could compromise the durability of the bond, whereas hydrophilicity and hydrolytic stability are considered antagonistic properties ${ }^{13,14}$.

There are theories explaining how water diffuses into a resin-based material after its application in an aqueous environment. An explanation of free volume theory reports that water can diffuse through polymer voids caused by resin-filler interfaces and morphological defects. Another possibility is that water molecules form hydrogen bonds with specific ionic groups in the polymer chain, causing water diffusion according to affinity ${ }^{16}$. Fabre et al. ${ }^{16}$ in 2007, compared the water sorption and solubility of different dentin bonding agents with respect to classification and light activation system, and the authors concluded that simplified systems were more susceptible to adverse effects of water and may become more prone to degradation over time independent of the type of light activation source.

Accordingly, Wei et al in 2011 investigated the kinetic process of water diffusion and mass change in five new resin matrix composites, and the samples were prepared following ISO 4049. Each resin matrix composite varied in water sorption and solubility cycles, which may affect clinical behavior. Therefore, this study reinforces the hypothesis that water sorption in resin composites depends on polymer (monomer, degree of conversion, polar interaction), charge (fraction, generic type, morphology, particle size, matrix dispersion), properties of the resin-filler interface, concentration of catalysts and initiators in the system and surface exposed to water. Thus, the polymer plays a dominant role in the water sorption in composite resin ${ }^{17}$. Soares et al. ${ }^{9}$ studied the microhardness of a dual-cured resin cement under the influence of thickness and color of a feldspathic ceramic. The authors concluded that good conversion of monomers depended on the quantity of light radiation that attained them. In spite of the present study having studied ceramics of the same color, different levels of translucency were tested, thus, the principle of influence on polymerization of the resin material could have been similar; that is, acting on the formation deficiently converted polymer networks, whose weak bonds favored water sorption and solubility of the material.

Runnacles et al. ${ }^{8}$ evaluated the influence of the thickness of ceramic restorations with different levels of translucency on the degree of conversion of a light activated resin 
cement, similar to the type used in the present investigation. The cement was light activated after interposition of ceramic restorations at low and high levels of translucency, of four thicknesses $(0.5 \mathrm{~mm} ; 1.0 \mathrm{~mm} ; 1.5 \mathrm{~mm}$ and $2.0 \mathrm{~mm})$. The authors concluded that the light activated cements must be used with caution under ceramics thicker than $1.5 \mathrm{~mm}$, due to the possibility of reduction in the degree of conversion with larger thicknesses.

In this study, a thickness of $1 \mathrm{~mm}$ was selected because it is commonly used when masking a darkend substrate is necessary. Faria and Silva in 2017 carried out an in vitro study to evaluate the light transmission of light curing units through ceramic cylinders and their effect on the polymerization kinetics of resin cement. In this study the ceramic ingots (IPS Empress Esthetic, shadow ET1) were sectioned to produce 0.5, 1.0 and $2.0 \mathrm{~mm}$ thick cylinders that were activated by two light curing units: SmartLite Focus and Valo Cordless. The authors concluded that total energy and irradiance decreased with increasing ceramic thickness in all cases ${ }^{18}$.

Another study by Martins et al in 2019 through a meta-analysis evaluated how the thickness variations in lithium disilicate ceramic restorations and the use of different light curing agents influence the degree of conversion of resin cements. The authors concluded that the thinner the ceramic material, the greater the degree of conversion. A thickness greater than $1.0 \mathrm{~mm}$ dramatically reduces the degree of conversion of double cured or photoactivated resins ${ }^{19}$.

The self-adhesive dual-cured cement exceeded ISO 4049 minimum requirements for cementing agents, in the three-time intervals (Water sorption $40 \mathrm{um} / \mathrm{mm}^{3}$; solubility $7.5 \mathrm{um} / \mathrm{mm}^{3}$ ) According to the ISO 4049 standards for water sorption and water solubility of polymer-based materials, water-sorption values under the limit of $40 \mathrm{mg} \mathrm{mm}$ and water-solubility values lower than $7.5 \mathrm{mg} \mathrm{mm}$ are considered acceptable ${ }^{20}$. The water sorption and solubility of self-adhesive dual cements was compared with those of other resin and ionomer cements in a previous study ${ }^{21}$, and the conclusion was that the they were more susceptible to water sorption. This finding was associated to their higher degree of acidity and hydrophilia, because these are the characteristics required in its process of bonding to dental structures ${ }^{21}$. Also, the acidic monomers from self-etching dual cements might negatively affect their degree of conversion through chemical interactions with the amine initiator.

The solubility of cements exceeded the iso requirements in most conditions tested. Furthermore, after 6 and 12 months, the light activated cement was found to present statistically higher solubility values than the others at all the levels of translucency. In the study of Jung et al. ${ }^{22}$, different sources of polymerization and modes of exposure were compared in the activation of a dual-cured resin cement through ceramic discs of different thicknesses: 1.0 and $2.0 \mathrm{~mm}$. The thickness of the restoration was found to act as a negative effect on the depth of conversion of the monomers, caused by the exponential reduction in light energy transmitted, called underpolymerization. This event could also have occurred in the light activate cement tested in this study, whereas, it depended exclusively on light to attain a good degree of conversion. 
The dual-cured resin cement presented statistically lower water sorption values in comparison with the other cementation agents, and low solubility values in comparison with the light activated cement in the storage time intervals tested. These results found could be justified due to the characteristic of addition of catalyzers in the chemical setting, which increased the degree of polymerization, because the conversion of monomers occurs even after use of the light source ${ }^{22}$. Braga et al. ${ }^{5}$ investigated the flexural strength; flexural modulus, and the hardness of four resin cements: Enforce (light activated), Variolink II (dual), RelyX ARC (dual) and C\&B (chemically activated), and verified the influence of the method of polymerization on the different properties of the material, and observed that dual-cured cements depend on light activation to reach higher degree of conversion values.

According to the limitations of the present in vitro study, the authors verified that the ceramic with the low degree of translucency had a negative influence on the passage of light, thereby elevating the loss and gain of water. However, the conventional dual cement - possibly due to the presence of the chemical setting propagated activation of the monomers even after polymerization and demonstrated more stable results when compared with the other cements, even after a long time of storage in water.

In a preliminary study, Leal et al. ${ }^{23}$ in 2016 evaluated the effect of the same levels of translucency of a laminated ceramic on the water sorption and solubility of three different adhesive cements (RelyX ARC, RelyX Veneer and Z350XT Flow). The authors observed that there was an inverse relationship between translucency of the ceramic and water sorption and solubility of the cementation agent in the immediate period (7 days of storage). Ceramics with higher levels of translucency produced acceptable water sorption and solubility values for the cementation agents studied. On the other hand, for ceramics with lower levels of translucency, the dual-cured resin cements or flowable resin composites must be preferred.

In view of these results, it would be necessary to seek an efficient way to compensate the attenuation of energy influenced by the translucency of the restorative material or for accentuating the activation of monomers. A prolonged period of activation by light could be used, as well as higher irradiation, up to $3500 \mathrm{~mW} / \mathrm{cm}^{2}$, or an increase in temperature of the agent. However, further studies are necessary to confirm the suitability, feasibility and clinical protocol of these procedures.

In conclusion, ceramic restorations with a low level of translucency may have a negative influence on the passage of light. The light activated resin, or conventional dual cementation agents are better indicated in these situations, because the chemical setting propagates activation of the monomers, thereby promoting good polymerization of the cement, acting on the longevity of the restoration even under the influence of time and humid environment of the oral cavity.

\section{CONFLICT OF INTERESTS}

The authors declare no potential conflict of interests with respect to the authorship and/or publication of this paper. 


\section{REFERENCES}

1. Guess PC, Schultheis S, Bonfante EA, Coelho PG, Ferencz JL, Silva, NR. All-Ceramic Systems: laboratory and clinical performance. Dent Clin North Am. 2011 Apr;55(2):333-52, ix. doi: 10.1016/j.cden.2011.01.005.

2. Uctasli S, Hasanreisoglu U, Wilson HJ. The attenuation of radiation by porcelain and its effect on polymerization of resin cements J Oral Rehabil. 1994 Sep;21(5):565-75.

3. Ilie N, Hickel R. Correlation between ceramics translucency and polymerization efficiency through ceramics. Dent Mater. 2008 Jul;24(7):908-14.

4. Cantoro A, Goracci C, Carvalho CA, Coniglio I, Ferrari M. Bonding potential of self-adhesive luting agents used at different temperatures to lute composite onlays. J Dent. 2009 Jun;37(6):454-61. doi: 10.1016/j.jdent.2009.02.006.

5. Braga RR, Cesar PF, Gonzaga CC. Mechanical properties of resin cements with different activation modes. J Oral Rehabil. 2002 Mar;29(3):257-62.

6. Souza-Junior EJ, Prieto LT, Soares GP, Dias CTS, Aguiar FHB, Paulillo LAMS. The effect of curing light and chemical catalyst on the degree of conversion of two dual cured resin luting cements. Lasers Med Sci. 2012 Jan;27(1):145-51. doi: 10.1007/s10103-010-0857-y.

7. ISO. International Standard. ISO 4049. Dentistry-Polymer-Based filling, restorative and luting materials. Geneva International Organization for Standardization; 2000. p. 18-21.

8. Runnacles P, Correr GM, Baratto Filho F, Gonzaga CC, Furuse AY. Degree of conversion of a resin cement light-cured through ceramic veneers of different thicknesses and types. Braz Dent J. 2014 Jan-Feb;25(1):38-42.

9. Soares CJ, Silva NR, Fonseca RB. Influence of the feldspathic ceramic thickness and shade on the microhardness of dual resin cement. Oper Dent. 2006 May-Jun;31(3):384-9.

10. Rasetto FH, Driscoll CF, Von Fraunhofer JA. Effect of light source and time on the polymerization of resin cement through ceramic veneers. J Prosthodont. 2001 Sep;10(3):133-9.

11. Gerdolle DA, Mortier E, Jacquot B, Panighi MM. Water sorption and water solubility of current luting cements: An in vitro study. Quintessence Int. 2008 Mar;39(3):e107-14.

12. Mese A, Burrow MF, Tyas M. Sorption and solubility of luting cements in different solutions Dent Mater J. 2008 Sep;27(5):702-9.

13. Reis AF, Giannini M, Pereira PNR. Influence of water-storage time on the sorption and solubility behavior of current adhesives and primer/adhesive mixtures. Oper Dent. 2007 Jan-Feb;32(1):53-9.

14. Aguiar TR,Andre CB, Ambrosano GMB, Giannini M. The Effect of Light Exposure on Water Sorption and Solubility of Self-Adhesive Resin Cements. Int Sch Res Notices. 2014 Oct 29;2014:610452. doi: 10.1155/2014/610452.

15. Alshali RU, Salim NA, Satterthwaite JD, Silikas N. Long-term sorption and solubility of bulk-fill and conventional resin-composites in water and artificial saliva. J Dent. 2015 Dec;43(12):1511-8. doi: 10.1016/j.jdent.2015.10.001.

16. Fabre HSC, Fabre S, Cefaly DFG, Carrilho MRO, Garcia FCP, Wanga L. Water sorption and solubility of dentin bonding agents light-cured with different light sources. J Dent. 2007 Mar;35(3):253-8.

17. Wei Y, Silikas N, Zhang Z, Watts DC. Diffusion and concurrent solubility of self-adhering and new resin-matrix composites during water sorption/desorption cycles. Dental Materials. 2011; 27 197-205.

18. Faria-e-Silva AL, Pfeifer CS. Effectiveness of high-power LEDs to polymerize resin cements through ceramics: An in vitro study. J Prosthet Dent. 2017 Nov;118(5):631-636. doi: 10.1016/j.prosdent.2016.12.013. 
19. Martins FV, Vasques WF, Fonseca EM. How the Variations of the Thickness in Ceramic Restorations of Lithium Disilicate and the Use of Different Photopolymerizers Influence the Degree of Conversion of the Resin Cements: A Systematic Review and Meta-Analysis. J Prosthodont. 2019 Jan;28(1):e395-e403. doi: 10.1111/jopr.12920.

20. Müller JA, Rohr N, Fischer J. Evaluation of ISO 4049: water sorption and water solubility of resin cements. Eur J Oral Sci. 2017 Apr;125(2):141-150. doi: 10.1111/eos.12339.

21. Chaves LP, Graciano FMO, Bim Junior O, Pedreira APRV, Manso AP, Wang L. Water interaction with dental luting cements by means of sorption and solubility. Brazilian Dental Science. 2013 Mar;15(4):29-35. doi: 10.14295/bds.2012.v15i4.826.

22. Jung H, Friedl KH, Hiller KA, Furch H, Bernhart S, Schmalz G. Polymerization efficiency of different photocuring units through ceramic discs. Oper Dent. 2006 Jan-Feb;31(1):68-77.

23. Leal CV, Queiroz APV, Foxton RM, Argolo S, Mathias P, Cavalcanti AN. Water Sorption and Solubility of Luting Agents Used Under Ceramic Laminates With Different Degrees of Translucency. Oper Dent. 2016 Sep-Oct;41(5):E141-8. 